\title{
sciendo
}

ISSN: $1231-4005$

e-ISSN: $2354-0133$

DOI: $10.2478 /$ kones-2019-0087

\section{MODELLING METHOD OF DYNAMIC CHARACTERISTICS OF MARINE THIN-WALLED STRUCTURE}

\author{
Do Van Doan, Adam Szeleziński, Lech Murawski, Adam Muc \\ Gdynia Maritime University, Faculty of Marine Engineering \\ Morska Street 83-87, 81-225 Gdynia, Poland \\ tel.: +48585586331 \\ e-mail:dodoan.vimaru@gmail.com,szelezinski1@wp.pl \\ l.murawski@wm.am.gdynia.pl
}

\begin{abstract}
Thin-walled structures are very popular in industries, especially in the field of shipbuilding. There are many types of equipment and structures of ships, which are made up of thin-walled structures such as hull, deck and superstructure. Therefore, the analysis and understanding of the static and dynamic characteristics of a thin-walled structure are very important. In this article, we focus on vibration analysis of a typical thin-walled structurerectangular plate, a basic structure of the hull. Vibration analysis of a rectangular thin plate is conducted by two methods: numerical modelling method of the finite element on Patran-Nastran software platform and experimental method implemented in the laboratory of Gdynia Maritime University. Thin rectangular plate is fixed one end by four clamping plates and is modelled with finite elements and different meshing densities. The numerical model of thin rectangular plate is divided into four cases. Case 1, thin rectangular plate, and clamping plates are modelled with two-dimensional elements. Case 2, the rectangular thin plate is modelled with two-dimensional elements; the clamping plates are modelled with three-dimensional elements. Case 3, both the rectangular thin plate and clamping plates are modelled with three-dimensional elements. Case 4, the rectangular thin plate, and clamping plates are modelled with three-dimensional elements with larger mesh density to increase the accuracy of the calculation results. After that, the results of vibration analysis according to the numerical modelling method on Patran-Nastran software platform for these cases were compared with the measurement results. From there, assess the accuracy of analysis results of selected numerical model methods and the ability to widely apply this numerical model method to other marine structures.
\end{abstract}

Keywords: Thin-walled structure, Vibration, FEM, rectangular plate, Patran-Nastran software

\section{Introduction}

Thin-walled structures are very popular in industries, especially in the shipbuilding industry. Thin-walled structures are described as mechanical structures, and their characteristics are the fact that one of the dimensions of the main structural components is much smaller than other dimensions. The thin-walled structure constitutes the key components that are important in the field of shipbuilding such as hull, deckhouses, and superstructures. For instance, the deck of a ship and the bulkhead grillages between cross girders are considered as thin plates with rectangular plates and stiffened beams. Therefore, it is very important to analyse the static and dynamic properties of rectangular thin plates. Good modelling method of single plates will allow us to reduce errors during analyses of whole ship hull and superstructure.

There have been many methods developed to analyse the static and dynamic properties of thinwalled structures as thin rectangular plates. In the past, some of the researchers have studied the natural vibration analysis of plates. Tanaka et al. [9] studied the integral equation approach for free vibration problems of elastic plate structures. This new integral equation approach and its solution give an approximate fundamental solution to the static problem. Wu and Liu [10] have been studied the new numerical solution technique called a differential cubature method for free vibration analysis of arbitrary shaped thick plates. 
Later researchers have developed the new methods free and forced vibration analysis of plate structures. Moon and Choi [4] have formulated the Transfer Dynamic Stiffness Coefficient method for Vibration Analysis of Frame Structures. Myung [7] has developed the Finite Element-Transfer Stiffness Coefficient Method free vibration analysis of plate structures. His approach is based on the combination of the modelling techniques in FEM and the transfer technique of the stiffness coefficient in the transfer stiffness coefficient method. Liew et al. [1] have investigated a meshfree Galerkin method free vibration analysis of unstiffened and stiffened corrugated plates. Their analysis carried on the stiffened corrugated plates, treated as composite structures of equivalent orthotropic plates and beams, and the strain energies of the plates and beams are added up by the imposition of displacement compatible conditions between the plate and the beams. The stiffness matrix of the whole structure was derived. Lu et al. [2] have investigated the differential quadrature method free vibration analysis of rectangular Kirchhoff plates with different boundary conditions. In case of complicated shapes generally, it is difficult to obtain an accurate analytical solution for structures with different sizes, various loads, and different material properties. Consequently, we need to apply on approximate numerical modelling methods for obtaining appropriate solutions of static and dynamic problems.

The current work deals with numerical modelling solutions implemented in Patran-Nastran software platform to obtain the natural frequency of a simply supported thin rectangular plate. Here, the numerical model of a thin rectangular plate is implemented in the software platform Patran - a pre-processor and post processing software. Computational analyses based on the finite element are performed in the Nastran software. By using the finite element, method stiffness and mass matrices were determined. These matrices are used to calculate the natural frequency of rectangular plate by solving the eigenvalue problem. The numerical results of the analysis are simply displayed as images in Patran software. This suggests that this current method can be successfully applied to free vibration analysis of any marine thin-walled structures.

\section{Vibration analysis of thin-walled plates}

According to Kirchhoff's classical [8] plate theory, the free vibration equation for the lateral motions of a homogeneous, isotropic, line is elastic thin plate is given by

$$
D\left(\frac{\partial^{4} z}{\partial x^{4}}+2 \frac{\partial^{4} z}{\partial x^{2} \partial y^{2}}+\frac{\partial^{4} z}{\partial y^{4}}\right)+\rho h \frac{\partial^{2} z}{\partial t^{2}}=0 .
$$

Here, $z(x, y, t)$ is the lateral displacement of the plate middle surface, with $x, y$-axes forming the plate, and $t$ is the time, $h$ is the thickness, $\rho$ is the mass density, $D=\frac{E h^{3}}{12\left(1-v^{2}\right)}$ is the plate stiffness factor, and $E$ and $v$ represent the Young modulus and Poisson ratio, respectively.

Suppose the principal mode of vibration of a thin plate is $Z(x, y)$; then the principal vibration can be written as follows:

$$
z(x, y, t)=Z(x, y) \exp (j \omega t)
$$

where $\omega$ is the natural frequency. When (2) is substituted into (1), the free vibration equation of thin plate can be expressed as follows:

$$
D\left(\frac{\partial^{4} Z}{\partial x^{4}}+2 \frac{\partial^{4} Z}{\partial x^{2} \partial y^{2}}+\frac{\partial^{4} Z}{\partial y^{4}}\right)-\rho h \omega^{2} Z=0 .
$$

Assume the following displacement function, which satisfies the boundary conditions, where $A_{m n}$ is an amplitude coefficient and $m$ and $n$ are integers.

$$
Z_{m n}(x, y)=A_{m n} \sin \left(\frac{m \pi x}{a}\right) \sin \left(\frac{n \pi y}{b}\right),
$$


where $a$ is the length of the plate, $b$ is the width of the plate.

After some transformation steps, the natural frequency of the thin plate are obtained as follows:

$$
\omega=\sqrt{\frac{D}{\rho h}}\left(\left(\frac{m \pi}{a}\right)^{2}+\left(\frac{n \pi}{b}\right)^{2}\right)
$$

The main vibration mode $Z(x, y)$ depends on the boundary conditions, as the plate is fixed.

The natural vibration frequency of the thin plate is calculated according to the theoretical formula illustrated in Tab. 1.

Tab. 1. Natural frequency for the thin plate according to the theory

\begin{tabular}{|c|c|c|c|}
\hline Vibration mode & Analytical $[\mathrm{Hz}]$ & FEM-3D det. [Hz] & Relative error [\%] \\
\hline 1 & 78.143606 & 50.45 & 35.439 \\
\hline 2 & 115.45148 & 132.86 & 15.079 \\
\hline 3 & 158.89779 & 151.40 & 4.719 \\
\hline 4 & 213.20566 & 188.19 & 11.733 \\
\hline
\end{tabular}

Experimental formulas are often formed based on experience and are conducted on many samples. However, with complex details and complex boundary conditions, empirical calculations often produce relatively large errors. As in this case, the relative error between empirical formula and the finite element method - FEM is greater than $35 \%$. Therefore, for complex structures that require high accuracy, the finite element method is a suitable choice.

\section{The numerical modelling of marine thin walled structures}

The numerical calculation was done with the help of the MSC Software package: PatranNastran $[5,6]$. These programs are based on finite element method. Natural vibration frequency of thin plates as well as the influence of numerical modelling on the accuracy of calculation results is determined. In this article, we calculate the thin plate by steel with the size $350 \times 220 \times 15 \mathrm{~mm}$, Young Modulus $E=21 \mathrm{GPa}$, density of the material $\rho=7850 \mathrm{~kg} / \mathrm{m}^{3}$, Poisson's ratio $v=0.3$ in four numerical models with the following boundary conditions:

- Model 2-D (two-dimensional) is modelled by two-dimensional finite elements, number of elements 2500 (Quad 4), number of nodes 2601, number of degrees of freedom 14022 as Fig. 1a,

- Model 2-3D, the thin plate is modelled with two-dimensional finite elements 2500 (Quad 4). The four clamping plates are modelled with three-dimensional elements 2100 (Hex8), number of nodes 4713, degrees of freedom 20358 as Fig. 1b,

- Model 3-D (three-dimensional), both thin plate and four clamping plates are modelled by threedimensional elements 4180 (Hex8), number of nodes 7314, degrees of freedom 21942 as Fig. 1c,

- Model 3-D det. has very large number of finite elements, 407586 elements (Hex8) to increase calculation accuracy, number of nodes 524264, degrees of freedom 1572795 as Fig. 1d.

Because the natural vibration of the cases is relatively similar, we only illustrated the natural vibration graphics of thin plates with the case of the 3-D det. The natural vibration of 3-D det. model is described in Fig. 2.

In fact, the natural vibration frequency of the ship is not too large, usually below $300 \mathrm{~Hz}$. It can be seen that vibration forms, starting with the sixth vibration mode, are very unlikely to resonate, with much higher frequencies (exceeding $300 \mathrm{~Hz}$ ). The results of calculating the natural frequencies of each model are presented in Tab. 2 and Fig. 3. 
a)

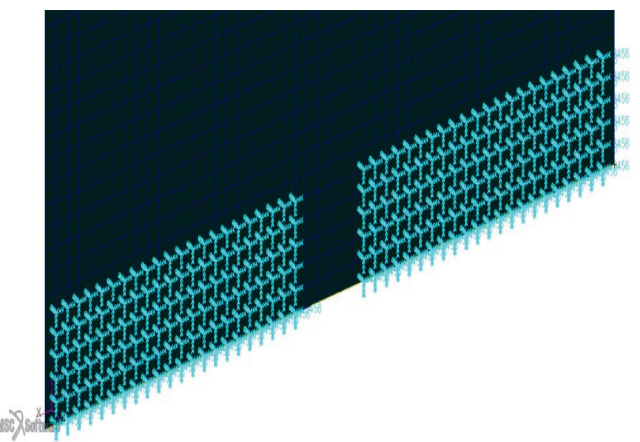

c)

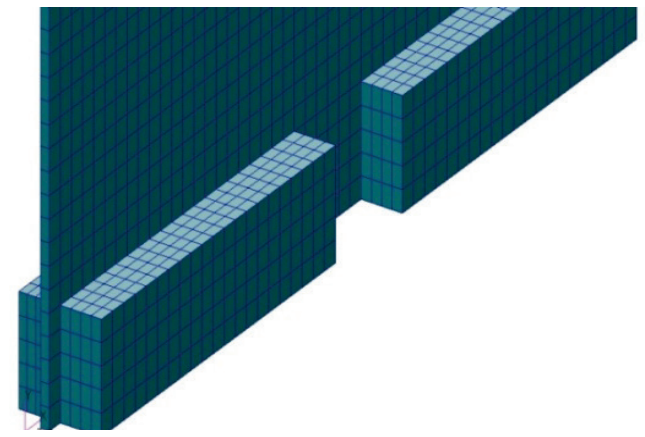

b)

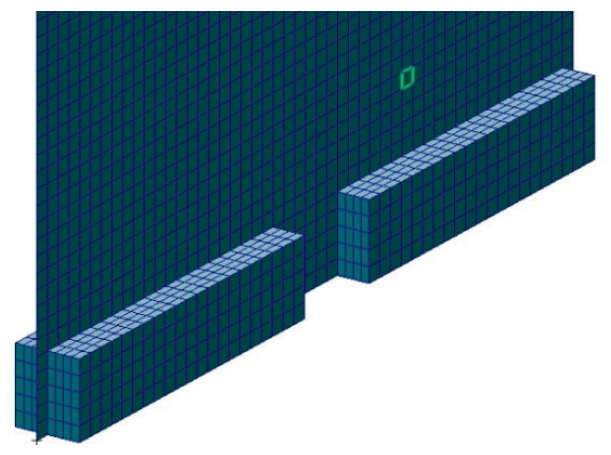

d)

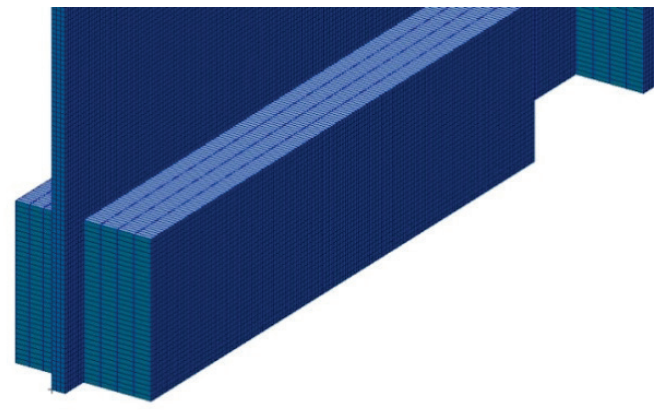

Fig. 1. Computational models of the thin plate, respectively: $2 D, 2-3 D, 3-D$ and 3-D det

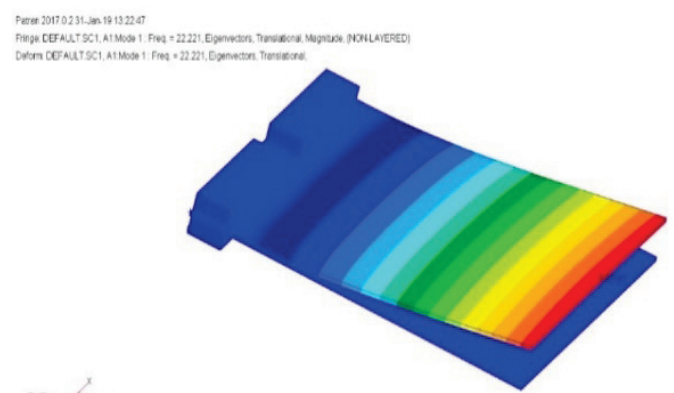

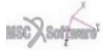

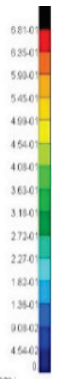

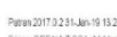

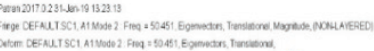

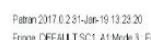

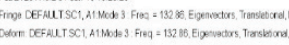

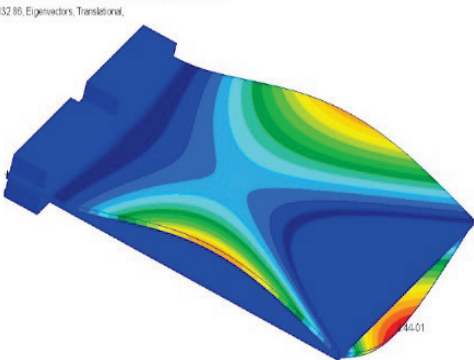

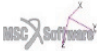

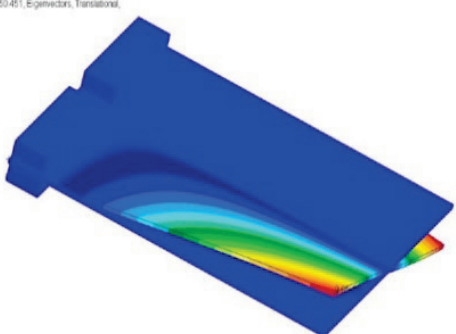

michesurinin

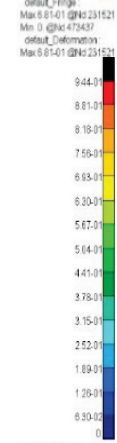

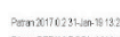

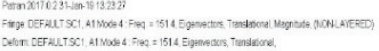

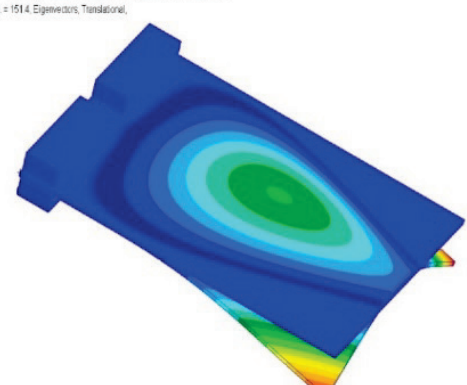

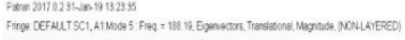
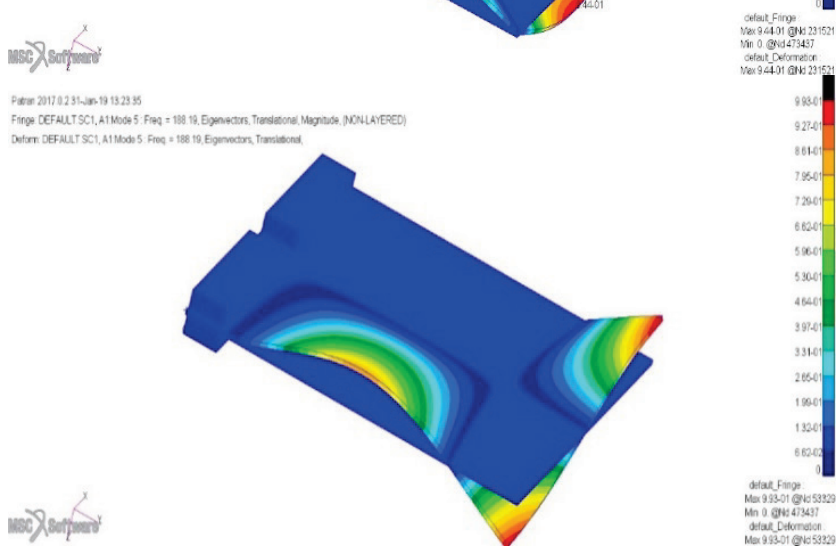

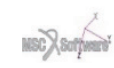

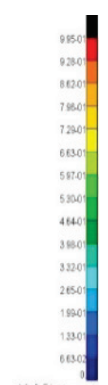

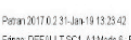

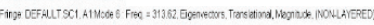

ate
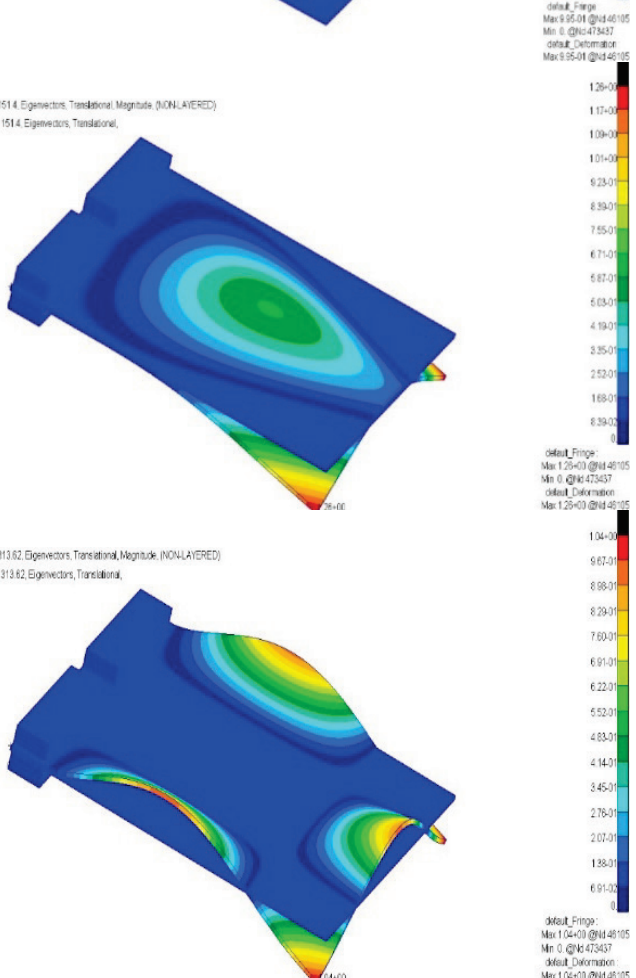

Fig. 2. Natural vibration modes of analytical thin plates 
Tab. 2. The natural frequencies of the calculation models

\begin{tabular}{|c|c|c|c|c|}
\hline \multirow{2}{*}{ Normal mode } & \multicolumn{4}{|c|}{ The natural frequency of the model $-f[\mathrm{~Hz}]$} \\
\cline { 2 - 5 } & Model 2D & Model 2-3D & Model 3-D & Model 3-D det. \\
\hline Mode No. 1 & 22.36 & 21.96 & 22.24 & 22.22 \\
\hline Mode No. 2 & 50.66 & 50.09 & 50.53 & 50.45 \\
\hline Mode No. 3 & 133.42 & 131.64 & 132.92 & 132.86 \\
\hline Mode No. 4 & 151.77 & 150.56 & 151.44 & 151.40 \\
\hline Mode No. 5 & 189.00 & 186.49 & 188.41 & 188.19 \\
\hline
\end{tabular}

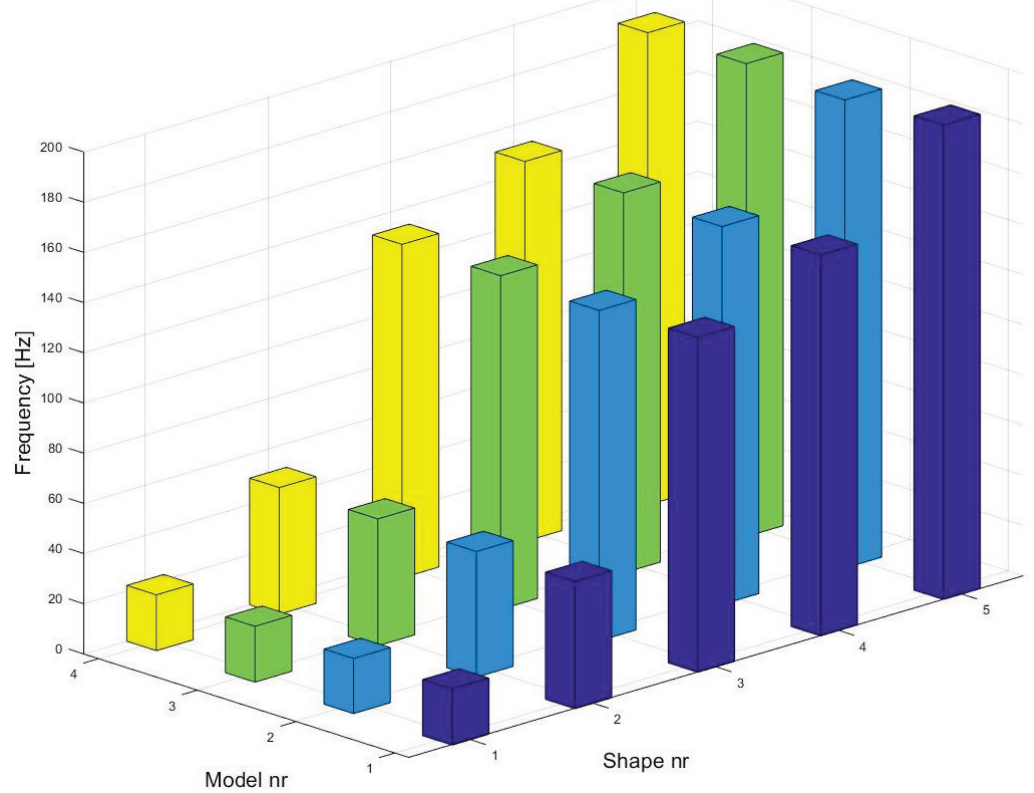

Fig. 3. The natural frequency of the analysed thin plate depending on the mathematical models

\section{The verify the results of numerical modelling by experimentally measured}

In this section, experiment with a real model of the thin plate; size $350 \times 220 \times 15 \mathrm{~mm}$ in steel material with boundary conditions as shown in Fig. 4.

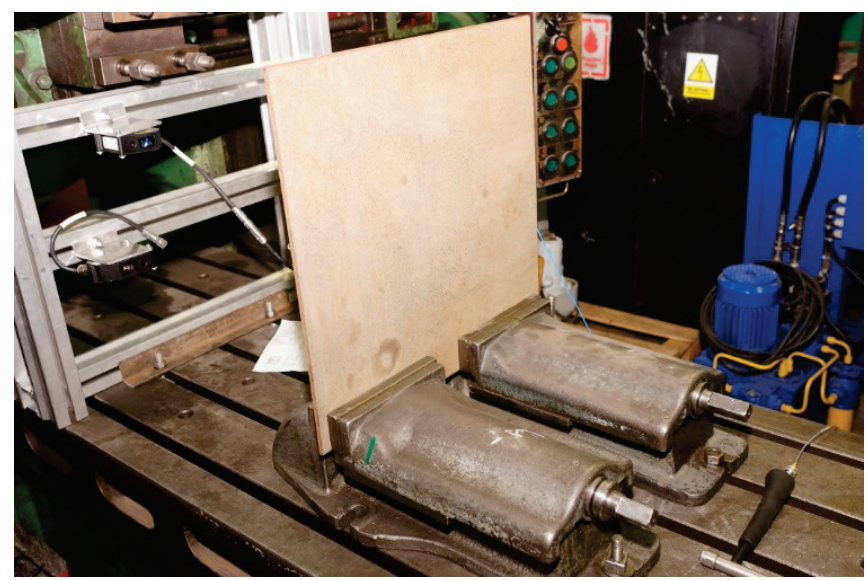

Fig. 4. Photograph of measuring model of thin plate

In tests, transmitters are attached according to the diagram as shown in Fig. 5. During the measurement of the real thin plate, three piezoelectric sensors were used A1, A2, and A3. During 
the study, the displacement of the plate was also registered with the use of laser sensors; however, the interpretation of the results is still unclear and requires further analysis, so they cannot be more detailed here.

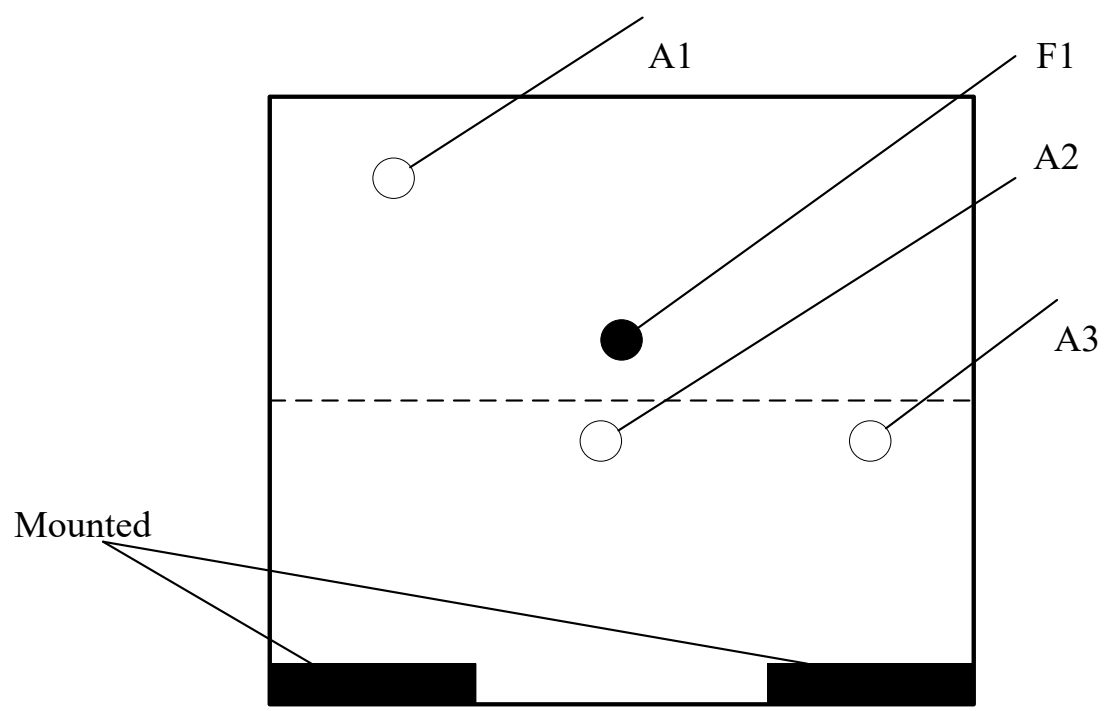

Fig. 5. Location of the transmitters during the second series of tests. A1-accelerometer (channel 1 - top) A2 - accelerometer (channel 2 - bottom centre), A3-accelerometer (channel 3 -bottom right) F1 - the place of impact with a modal hammer

The vibration parameters obtained by the results of the measurements are carried out on the actual thin plate with the above boundary conditions. All tests have been done according to good practice principles. They include calibration of the apparatus used to increase the accuracy of the measurement. The number of repetitions of the results obtained in the pre-defined frequency domain is large enough to meet the accuracy requirement (the lack of repeatability may indicate incorrect assembly of the test subject). The frequencies in the measurement tests were determined with a resolution of $0.125 \mathrm{~Hz}$, allowing relative measurement errors of up to $0.6 \%$ for the first natural frequency. It should be emphasized that all computational errors lower measurement errors should be considered as 0 . The vibration signals are performed Fourier-FFT fast analysis in MATLAB software to obtain the accelerated spectral response of vibration [3]. On the graph, the acceleration response of the vibration determines the natural frequency values at the vertices of the graph. The response of the acceleration spectrum vibrates with the forced modal hammer of the thin plate is illustrated in Fig. 6.

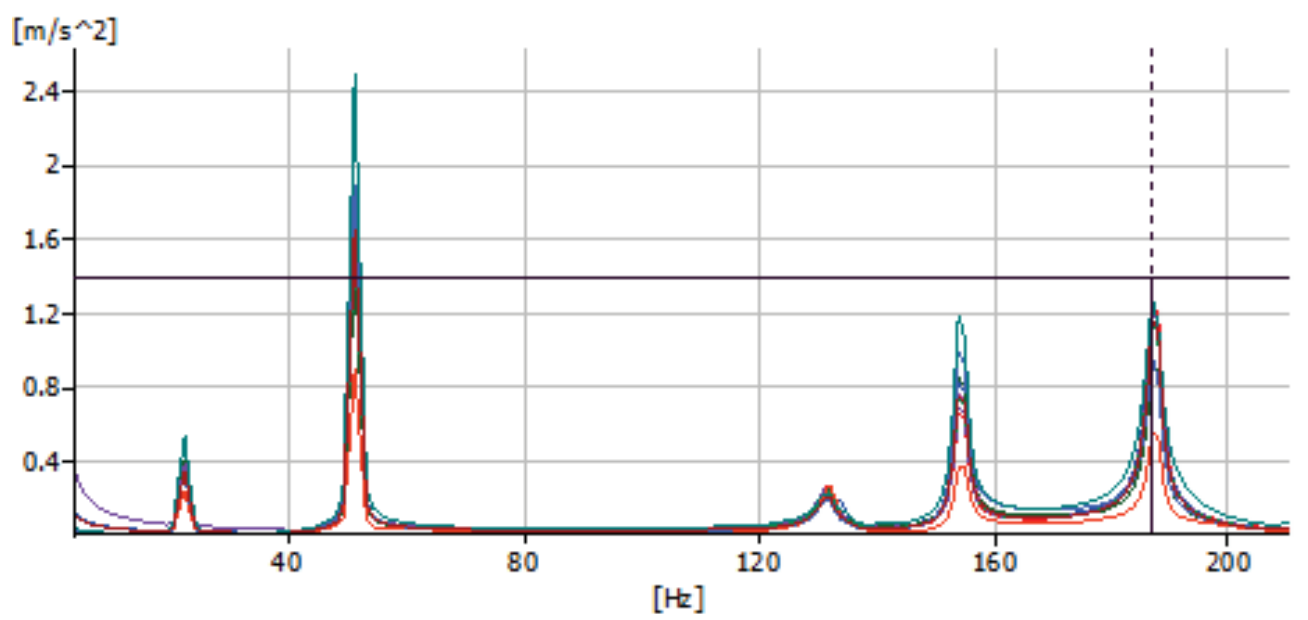

Fig. 6. The response of the acceleration spectrum vibrates with the forced modal hammer of the thin plate 
In Fig. 6, the approximation of the spectra of the thin plate is presented, thanks to which it is possible to read the value of the mode of the natural vibrations of the thin plate. They are $21.875 \mathrm{~Hz}, 51.125 \mathrm{~Hz}, 132 \mathrm{~Hz}, 154.125 \mathrm{~Hz}$, and $187.5 \mathrm{~Hz}$, respectively.

\section{Conclusions}

The natural frequency results obtained by numerical model analysis - finite element method implemented on Patran-Nastran software and experimental methods are presented in Tab. 3.

Tab. 3. The natural frequencies of calculation models and measurement

\begin{tabular}{|c|c|c|c|c|c|}
\hline & \multicolumn{4}{|c|}{ The natural frequency of the model: [Hz] } & \multirow{2}{*}{$\begin{array}{c}\text { Measurement } \\
\text { tests [Hz] }\end{array}$} \\
\cline { 2 - 5 } & Model 2D & Model 2-3D & Model 3-D & Model 3-D det. & 21.875 \\
\hline Mode No. 1 & 22.36 & 21.96 & 22.24 & 22.22 & 51.125 \\
\hline Mode No. 2 & 50.66 & 50.09 & 50.53 & 50.45 & 132.00 \\
\hline Mode No. 3 & 133.42 & 131.64 & 132.92 & 132.86 & 154.125 \\
\hline Mode No. 4 & 151.77 & 150.56 & 151.44 & 151.40 & 187.500 \\
\hline Mode No. 5 & 189.00 & 186.49 & 188.41 & 188.19 & \\
\hline
\end{tabular}

Correlation error between natural frequencies obtained by numerical modelling and natural frequencies obtained by the empirical method is described in Tab. 4 and Fig. 7.

Tab. 4. Relative natural frequency error between measurement method and numerical modelling method

\begin{tabular}{|c|c|c|c|c|}
\hline \multirow{2}{*}{} & \multicolumn{4}{|c|}{ Relative errors \% } \\
\cline { 2 - 5 } & Model 2D & Model 2-3D & Model 3-D & Model 3-D det. \\
\hline Mode No. 1 & 2.17 & 0.39 & 1.64 & 1.55 \\
\hline Mode No. 2 & 0.92 & 2.07 & 1.18 & 1.34 \\
\hline Mode No. 3 & 1.06 & 0.27 & 0.69 & 0.65 \\
\hline Mode No. 4 & 1.55 & 2.37 & 1.77 & 1.80 \\
\hline Mode No. 5 & 0.79 & 0.54 & 0.48 & 0.37 \\
\hline
\end{tabular}

The relative errors between numerical modelling method and measuement method

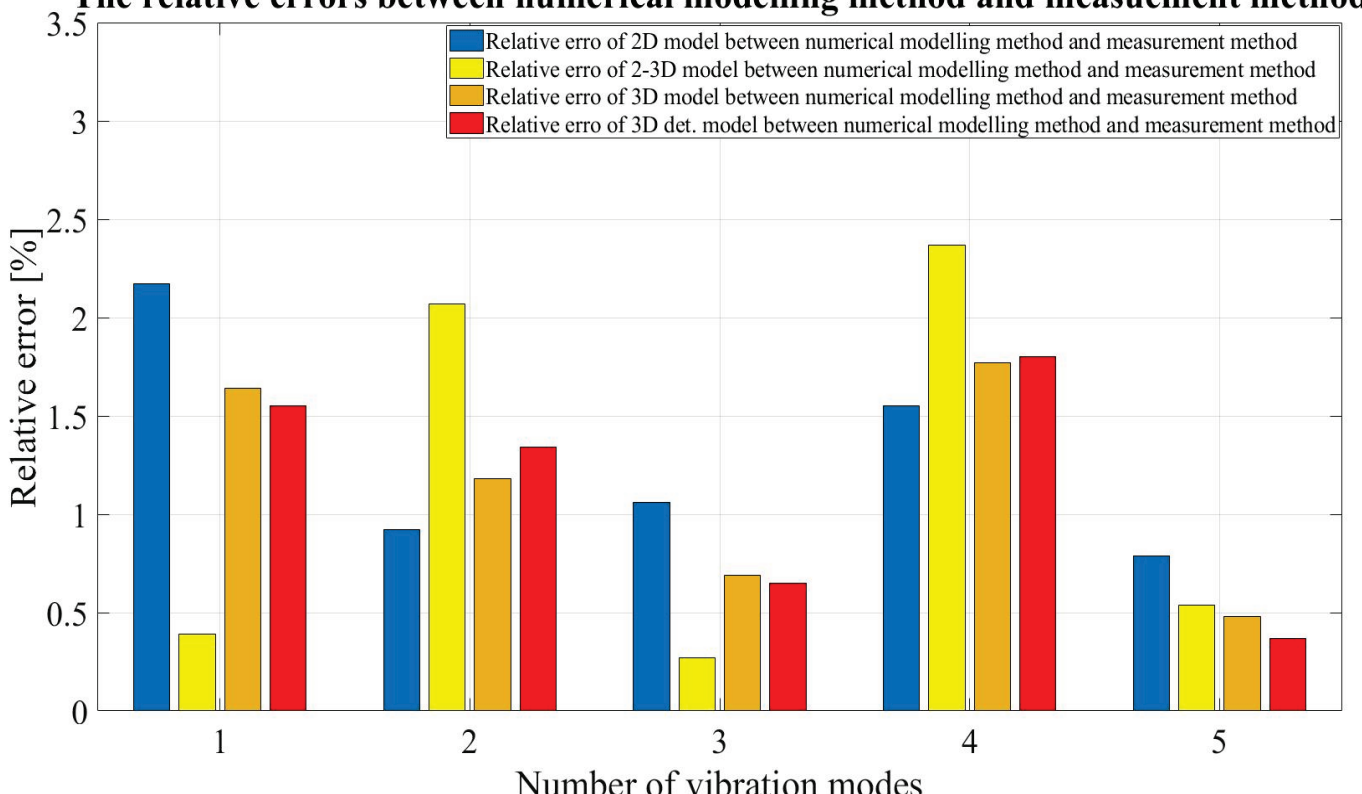

Fig. 7. Relative errors of calculations of the natural frequency of the analysed thin plate 
The results of calculating the natural frequencies of the individual models together with the comparison with the measurement results are shown in Tab. 4. Computational errors (related to measurement tests) and numerical modelling methods are shown in Fig. 7. All numerical models, including the simplest 2-D model, perfectly reflect the dynamic characteristics of the actual plate, although each model has its own disadvantages. First, considering the 2-D model, the basic plate model is most often used with selected boundary conditions suitable for constraints, respectively. This model may be too simple accurately to reproduce the dynamic properties of thin plates. In particular, boundary conditions have a large impact on the accuracy of the results. The simplest 2-D model does not take into account the rigidity of the mounting plate. Therefore, the relatively large error of calculations occurs for the simplest 2-D model and for the first natural vibration form is $2.17 \%$. In the $2-3 \mathrm{D}$ model, problems occur when connecting plate elements to solid elements; plate elements are characterized by six degrees of freedom on each node, while solid elements do not include rotation degrees of freedom (three degrees of freedom for each node). These results in a relatively large error for vibration form (second and fourth) related to twisting, relative errors of $2.07 \%$ and $2.37 \%$, respectively. The disadvantage of the $3 \mathrm{D}$ model is completely the weak expression of the deformation distribution on the thickness of the plate due to its linear shape function and an element on each sheet thickness. The exact 3D det. model does not have any of the above disadvantages; however, it is many times larger than other models. It should be noted that the model 3-D det. has more than 1.5 million degrees of freedom. A 3-D det model cannot analyse without using the connected supercomputer in the network. A significant increase in computing costs does not include the increased accuracy of the calculation. Therefore, 2-3D model is evaluated as optimal according to the viewpoint of technical practice. The measurement method has additionally verified and proved the correctness of the selected 2-3D model method.

\section{References}

[1] Liew, K. M., Peng, L. X., Kitipornchai, S., Vibration analysis of corrugated Reissner Mindlin plates using a mesh-free Galerkin method, International Journal of Mechanical Sciences, Vol. 51, Iss. 9-10, pp. 642-652, 2009.

[2] Lu, C. F., Zhang, Z. C., Chen, W. Q., Free vibration of generally supported rectangular Kirchhoff plates: State-space-based differential quadrature method, International Journal for Numerical Methods in Engineering, Vol. 70, pp. 1430-1450, 2007.

[3] MATLAB Software, MATLAB the language of Technical Comptuting, The MathWorks, Inc. 3 Apple Hill Drive Natick, MA 01760-2098.

[4] Moon, D. H., Choi, M. S., Vibration Analysis for Frame Structures Using Transfer of Dynamic Stiffness Coefficient, Journal of Sound and Vibration, Vol. 234, Iss. 5, pp. 725-736, 2000.

[5] MSC.Software, MSC Nastran 2018, Quick Reference Guide, MSC.Software Corporation, Santa Ana, CA 92707, USA 2017.

[6] MSC.Software, MSC Nastran 2017, Dynamic Analysis User's Guide, MSC.Software Corporation, Santa Ana, revision 0, CA 92707, USA 2016.

[7] Myung, S. C., Free Vibration Analysis of Plate Structures Using Finite Element-Transfer Stiffness Coefficient Method, KSME International Journal, Vol. 17, No. 6, pp. 805-815, 2003.

[8] Petyt, M., Introduction to Finite Element Vibration Analysis, Cambridge University, New York 1990.

[9] Tanaka, M., Yamagiwa, K., Miyazaki, K., Ueda, T., Free vibration analysis of elastic plate structures by boundary element method, Engineering Analysis, Vol. 5, Iss. 4, pp. 182-188, 1988.

[10] Wu, L., Liu, J., Free vibration analysis of arbitrary shaped thick plates by differential cubature method, International Journal of Mechanical Sciences, Vol. 47, pp. 63-81, 2005.

Manuscript received 08 August 2019; approved for printing 09 December 2019 